\title{
Ciudades amazónicas intermedias, pesca y fronteras
}

\section{Cidades médias amazônicas, pesca e fronteiras}

Yohana Pantevis - Historiadora, Mestre en Estudios Amazónicos por la Universidade Nacional de Colombia e Doutoranda en Estudios Amazónicos por la Universidad Nacional de Colombia., Sede Amazonia.

Germán Palacio - PhD Historia; Profesor Titular de la Universidad Nacional de Colombia, Sede Amazonia.

\section{Resumo}

Leticia, Amazonas, Colombia, durante la década de 1950 de apariencia aldeana, vivió un importante proceso de crecimiento y modernización generado principalmente por las inversiones del Estado, argumenta Jorge Picón (2010). Yohana Pantevis (2013) propuso que el desarrollo de actividades comerciales permitió el surgimiento y consolidación del sector privado como un factor explicativo clave al igual que el Estado. Este artículo, apunta a encadenar estas dos explicaciones. Muestra que una combinación de Estado con inversiones de un emergente sector privado dio lugar a una fuerte actividad económica que ha definido, en buena medida, el desarrollo de la ciudad. Lo que de alguna forma contradice las teorías que sostienen que la ausencia de Estado es característico de los territorios de frontera; contradice las tesis neoliberales sobre que el mercado y el sector privado son los agentes de desarrollo y que el Estado debe reducirse a su menor expresión. Aquí se ilustra este argumento de engarzamiento entre lo público y lo privado, con el desarrollo urbano de ciudades intermedias, utilizando el caso de Leticia, Amazonas.

\section{Palavras-chave}

Historia. Pesca. Frontera. Estado y mercado.

\begin{abstract}
Letícia, Amazonas, Colômbia, de aparência aldeã durante a década de 1950, viveu um importante processo de crecimento e modernização, gerado principalmente pelos investimentos do Estado, argumenta Jorge Picón (2010). Yohana Pantevis (2013) propus que o desenvolvimento de atividades comerciais permitiu o surgimento e consolidação do setor privado, como um fator explicativo chave ao igual que o do Estado. Este artigo procura encadear estas dois explicações. Mostra que uma combinação de Estado e investimentos de um emergente setor privado deu lugar a uma forte atividade econômica que tem definido, em boa medida, o desenvolvimento da cidade. O que de alguma forma contradiz as teorias que sustentam que a ausência do Estado é caraterístico dos territórios de fronteira; contradiz as teses neoliberais de que o mercado e o setor privado são os agentes de desenvolvimento e que o Estado deve se reduzir a sua menor expressão. Ilustra-se aqui este argumento de entroncamento entre o público e o privado, com o desenvolvimento urbano de cidades médias, utilizando o caso de Letícia, Amazonas.
\end{abstract}

\section{Keywords}

História. Pesca. Frontera. Estado y mercado 


\section{INTRODUCCIÓN}

Desde el conflicto con el Perú en la década de 1930, al poblado de Leticia se le reconoció una importancia geopolítica en la frontera de Colombia con Perú y Brasil. Leticia es una ciudad-puerto sobre el río Amazonas que surgió y se desarrolló a través de la acción del Estado y el comercio'. Después de la segunda mitad del siglo XX la actividad mercantil más importante, junto con la exportación de pieles y animales, y dejando de lado la economía ilegal de la coca, que serán motivo de estudio en otro artículo, fue y ha sido la comercialización de pescado. Dicha actividad fue desarrollada inicialmente por comerciantes provenientes de la zona andina del país, quienes vieron en este recurso una mercancía promisoria que servía como carga de compensación, es decir, carga para garantizar la rentabilidad de los vuelos comerciales en la ruta Leticia-Bogotá. Es importante destacar que esta actividad trajo consigo una transformación y una mediana tecnificación en el proceso de explotación que es descrita por los pescadores como "más peces, en menos tiempo y esfuerzo".

Aunque la quina y el caucho son mercancías emblemáticas del extractivismo amazónico, evidentemente, la pesca es una de las actividades más antiguas de la Amazonia y en las últimas décadas se ha convertido en una importante actividad comercial para Leticia y otras poblaciones del departamento del Amazonas. Con una presión demográfica baja, la cantidad de pescado es clave para alimentar una población ribereña y también para generar excedentes. Adicionalmente, con el descubrimiento de la biodiversidad a fines del siglo XX, expertos señalan la importancia de la diversidad íctica. Este potencial de la cuenca amazónica ha sido ampliamente reconocido y para el año 2006 se calculó que existen por lo menos 2.000 especies $^{3}$, de las cuales se reportan al menos 94 especies de interés económico; 31 se comercializan como ornamentales; 50 como peces de consumo; y 13 especies son destinadas para ambos fines (SALINAS; AGUDELO, 2000). Sin embargo, la explotación comercial se concentra sólo en el 5\% de las especies existentes. Esta riqueza íctica está condicionada por tiempos de abundancia y de escasez, en donde solo el conocimiento del comportamiento de los elementos que la conforman -la tierra, el bosque y el río- permitirían satisfacer casi por completo las necesidades de la población que la habita (WITKOSKI, 2007).

1 Para mayor información sobre la fundación de la ciudad de Leticia ver: PICÓN, J. (2010); NAVIA, G. (1994).

2 Entrevista realizada a pescadores y miembros de ASOPESCAM en Leticia 2012.

3 La cuenca del río Amazonas es la más grande del mundo $\left(7.500 .000 \mathrm{~km}^{2}\right)$ y desde una perspectiva ecológica es un sistema pulsante altamente productivo, principalmente por el aporte periódico de sedimentos ricos en nutrientes que son arrastrados desde la cordillera de los Andes por las aguas del río Amazonas. Ver: GALVIS et al, (2006). 
En su libro Violencia politica en Colombia: de la nación fragmentada a la construcción del Estado, el historiador Fernán González (2002) propone que el Estado (colombiano) se construye en tiempos y espacios temporalmente diferenciados o, mejor, decimos nosotros, no sincronizados. Germán Palacio (2006) ha propuesto que la historia amazónica es asincrónica y excéntrica para reflejar esa diferenciación en tiempo y espacio. Es curioso y hasta paradójico que, muchas veces, lugares de la Amazonia son considerados lugares remotos desconectados del mundo y preservados de la civilización occidental, cuando una característica histórica clave de buena parte de la Amazonia es que su conexión con el mundo es más fuerte o recurrente que con la Nación. Por ello se puede decir que la Amazonia ha hecho parte de la interconexión global desde los viajes de Francisco de Orellana en 1538 (PALACIO, 2008).

Dejando de lado grandes y populosas ciudades amazónicas tales como Belem do Pará, fundada en el siglo XVII; Manaos, siglo XVIII e Iquitos, siglo XIX, todas consolidadas en épocas de la expansión mundial del caucho y la seringa desde fines del siglo XIX, recientemente estudios de ciudades intermedias en la Amazonia han empezado a llamar la atención, máxime que su crecimiento demográfico ha estado despuntando en los últimos tiempos y el interés por estudiarles es creciente (ANGEOLETTTO; ESSY; RUIZ, 2016).

Este estudio incorpora elementos claves de la historia de la ciudad de Leticia, propuesto por Jorge Picón (2010) y que atribuye al Estado la transformación de una aldea en ciudad sobre la base de inversiones del Estado colombiano en la época del General Gustavo Rojas Pinilla, caudillo y líder militar en los años 1950s en Colombia. Avanzando sobre esta idea, Yohana Pantevis (2013) ha mostrado que la historia de la pesca, abanderado por el sector privado, fue igualmente clave para este proceso. De una parte, el Estado aportó sustancialmente al desarrollo urbano de la ciudad conectándola con la capital del país, a través de la construcción del aeropuerto para hacer posible el comercio de pieles, de otra, el comercio de pescado de Leticia a Bogotá ha sido jalonado por el sector privado, para lo cual se requirieron traer aviones comerciales, con lo cual, los dos sectores se retroalimentan. En síntesis, el desarrollo del capitalismo naciente en la región amazónica está atado al papel clave del Estado y de un sector privado que aprovecha lo realizado por el Estado. Por razones históricas de carácter geopolítico, una población de enclave se fue consolidando desde la década de los 1950's. La relación entre la ciudad y la pesca puede servir de elemento clave para el análisis de las ciudades pequeñas y medianas en el medio Amazonas. La trasformación de la "frontera" en "región" implica el desarrollo de ciudades capitales, como es el caso de Leticia. 


\section{EL NACIMIENTO DE UNA CIUDAD DE SELVA}

Leticia es una ciudad-puerto sobre el río Amazonas, 4 grados sur, el punto más meridional de Colombia en la selva amazónica. Fue fundada por el capitán peruano Benigno Bustamante el 25 de abril de 1867 como el fuerte "Gran Mariscal Ramón Castilla” o San Antonio como parte del proceso de consolidación peruano sobre la Amazonia y década y media antes de la Guerra del Pacífico que resultó en la derrota de Perú y Bolivia ante Chile y que, probablemente, desvió la atención de la elite peruana de sus intentos de consolidar sus avances territoriales sobre la Amazonia a costa de las aspiraciones de Ecuador y Colombia. El 15 de diciembre de 1867, Manuel Charón, ingeniero de la Comisión Hidrográfica del Amazonas, cambió el nombre del puerto por el de Leticia; según la historia popular, en honor a una joven de familia anglo-peruana llamada Leticia Smith Buitrón (PANTEVIS, 2015). Con el paso de los años el puerto fue convirtiéndose en un punto de comercio fluvial y en 1900 el estado peruano creó la "Aduanilla" para controlar el tráfico de caucho. A principios del Siglo XX y tras la firma del Tratado Lozano-Salomón entre Colombia y Perú en 1922, a través del cual se traza el así conocido como Trapecio Amazónico, cediendo Perú a Colombia la soberanía sobre Leticia a través del cual el estado colombiano adquirió una salida al río Amazonas y el estado peruano se hizo acreedor formal y oficial de una frontera hasta la margen derecha del río Putumayo (GONZÁLEZ-PEÑA; SAMACÁ, 2012, p. 376). Enseguida, se crea la Comisaria Especial de Amazonas, artículo $3^{\circ}$ de la Ley 96 de 1928, aunque se trata de la organización de un territorio que permanece, como muchos otros territorios de Colombia en la Amazonia y en la Orinoquia y el extremo norte del Caribe en la Guajira, en una especie de estatus colonial interno administrado por el Estado nacional desde Bogotá, como una parte de lo que se conocía como "territorios nacionales".

En 1930 arribó a Leticia el coronel Luis Acevedo en el vapor "Nariño", designado para recibir los territorios que pasarían a ser parte del territorio nacional por parte de Perú a Colombia, trayendo consigo al primer Comisario del Amazonas, el señor Abdón Villareal, al padre Bartolomé de Igualada, y a 32 civiles más para repoblar con nacionales colombianos la ciudad (RUIZ, 2001), ya que para ese momento la mayoría de la población era de origen peruano (ZÁRATE, 2008). Se trazaron las calles siguiendo el modelo español de cuadrícula, el modelo ortogonal de la ciudad colonial, que facilitó la adjudicación gratuita de los primeros lotes a los colonos que llegaron a la ciudad.

En septiembre de 1932, un grupo de ciudadanos peruanos, habitantes del vecino departamento de Loreto, con un fuerte arraigo identitario, irrumpieron 
en Leticia dando inicio al conflicto colombo-peruano que finalizó en mayo de 1934, generando una ruptura entre el borroso pasado colonial amazónico y la inmersión de la ciudad en la lógica institucional del estado colombiano. En el momento que ocurrió el asalto a Leticia, el contexto de la Gran Depresión de fines de los años 20’s presiono a la baja el café y el petróleo, y generó la caída de los precios agrícolas; junto con unos ingresos fiscales reducidos que complejizaban el escenario nacional, acabaron por dar al traste con la hegemonía conservadora construida desde la década de 1880 s con la promulgación de una Constitución fuertemente centralista que perduró por cien años.

Cuando llegó a Bogotá la noticia de que Leticia había sido invadida por los peruanos, loretanos (Loreto es el departamento peruano contiguo a Amazonas) en sentido más estricto, no necesariamente apoyados por el gobierno central en Lima, las elites colombianas se olvidaron por un momento de las cuestiones económicas y políticas internas y el nuevo gobierno liberal pidió apoyo de toda la ciudadanía para confrontar esta situación. Una vez solucionado el conflicto, sobre el cual este artículo no se detiene ${ }^{4}$, se precisaron los límites fronterizos y se sentaron algunas bases para intentar consolidar las líneas fronterizas internacionales de Colombia que, en buena medida se lograron, con excepción de los casos disputados entre Colombia y Venezuela, así como con Nicaragua que persisten hasta hoy en día.

La ubicación geográfica de la ciudad se enmarca en un punto de encuentro que da frente a Brasil, Perú y Colombia. Desde un contexto nacional se trataba de una ciudad de enclave a la cual sólo se accede a ella por vía aérea desde la ciudad de Bogotá, o fluvial por el río Putumayo hasta llegar por el Içá, Brasil, y regresándose por el Amazonas que los brasileros llaman Solimões.

Tras el conflicto colombo-peruano, la ciudad es percibida por los colombianos bajo el lente de importancia simbólica y geopolítica, aunque desconectada del resto del país, poco a poco consolida una importante estructura urbana regional "tanto con funciones de control territorial, de los flujos comerciales y humanos, así como enlace comercial entre los estados limítrofes" (APONTE, 2011, p. 34). Avanzando los años 1950s, bajo una política de apropiación de las fronteras lideradas por el General Rojas Pinilla, se iniciaron varias obras públicas con el fin de mejorar la infraestructura básica en cuanto a servicios públicos domiciliarios, educación, comunicación y transporte, hasta ese entonces casi que inexistentes (PICÓN, 2010). Sin embargo, el papel del Estado para delimitar los bordes internacionales y construir un poblado de enclave son insuficientes para comprender el proceso de consolidación de Leticia. La quina y

4 Para mayor información sobre el conflicto ver: DONADIO (2002). 
el caucho, bastante estudiados pueden ser los más importantes antecedentes de las economías extractivistas de la región. Sin embargo, en el siglo XX, el comercio de piles y de fauna, primero y, luego, la comercialización de pescado son claves para la consolidación de la ciudad.

\section{EL PAPEL DE LAS PIELES Y LA FAUNA SILVESTRE PARA LA CONSOLIDACIÓN DE UNA CIUDAD DE ENCLAVE}

Los primeros pasos de producción industrial se dan con la fábrica de bebidas gaseosas "El Progreso", fundada en 1938 por Don Antonio Perea consolidándose a nivel local y fronterizo (PICÓN, 2010, p. 68). Durante la década de 1950 se evidencia un incremento del sector privado con el surgimiento de casas comerciales, tiendas y panaderías. Sin embargo, lo que verdaderamente empieza a cambiar significativamente la situación es que a mediados del siglo XX surgió un importante comercio de pieles y de fauna silvestre en la región amazónica; las pieles eran utilizadas en la elaboración de bolsos, chaquetas, sombreros y zapatos, comercializados principalmente en Europa y Estados Unidos; mientras que la fauna silvestre era comercializada en zoológicos, universidades y tiendas de mascotas en Estados Unidos principalmente ${ }^{5}$. Probablemente, la industria del entretenimiento de Florida y del cinturón del sol en los Estados Unidos también juega un papel importante. Esta nueva bonanza extractiva revive en los colonos asentados en la ciudad la posibilidad de impulsar el comercio y los medios de transporte, siendo la extracción de fauna silvestre y pieles un dinamizador de la economía local y generador de ingresos que prometía, para algunos, como se decía en la época, traer el tan anhelado progreso. La gran demanda externa de fauna silvestre y pieles permitió que la aviación comercial en la ciudad fuera liderada por el sector privado que necesitaba enviar al exterior del país una parte de los recursos naturales existente en la región.

Desde 1941 se había establecido una línea semanal de servicio aéreo en la ruta Bogotá - Leticia en el hidroavión PBY Catalina, pero este avión era bastante lento y tenía poca capacidad de carga, limitando el crecimiento de la actividad comercial. Luego, los comerciantes locales empezarán a recordar con gran gratitud al militar Rojas Pinilla, quién antes de ser presidente se desempeñó como director de la Aeronáutica Civil y se interesó en unir al país a través del

Palabras de Mike Tsalickis, propietario de la empresa Tarpoon Zoo. Entrevista concedida a Germán Palacio en Tampa - Florida, 13 de marzo de 2013. 
proyecto "Pistas de aterrizaje en Colombia" que pretendía además de Leticia conectar otras regiones apartadas con el interior del país, a fin de poder ejercer la soberanía nacional (ESCOBAR, 2008). Fue así como en 1950 se construyó en Leticia una rudimentaria pista de 900 metros de longitud que permitió el transporte de pasajeros y carga en aviones con ruedas (ESCOBAR, 2010). Así, asegurar la soberanía nacional es combinada con el impulso del comercio de una ciudad de enclave en la frontera amazónica.

En 1955 se iniciaron las obras para construir un aeródromo que recibiría aviones de mayor capacidad de carga, apoyado por el Ministerio de Guerra, como se llamaba en la época el actual Ministerio de Defensa, y el Departamento de la Aeronáutica Civil. La pista original fue prolongada hasta 1.300 metros, lo que permitió la operación de aviones DC-4 y su construcción fuera encomendada a la Empresa Colombiana de Aeródromos, quién elaboró los estudios topográficos, el diseño de la pista y del edificio que conformarían el aeropuerto. Ese mismo año se inició el servicio del aeropuerto Alfredo Vásquez Cobo, recordando al general conservador de la época de la Guerra con el Perú, con la exoneración del pago a las compañías aéreas por los servicios de aterrizaje y radio-ayudas, según Resolución Reglamentaria No. 12 de 1955, emitida por la Empresa Colombiana de Aeródromos - ECA, como un incentivo a las empresas aéreas nacionales e internacionales para garantizar la prestación del servicio en la región.

El argumento del Estado para justificar la financiación y construcción del aeropuerto era la urgente necesidad de proveer a la región de mercancías desde el interior del país ya que estaba desprovista de bienes de consumo intermedios para los nuevos pobladores colonos y funcionarios estatales. Esta situación facilitó la exportación de fauna silvestre y pieles, junto con la comercialización de maderas finas que fueron trasportados de Leticia a Bogotá como carga de compensación.

Leticia tuvo una significativa participación en este comercio, destacándose junto con Barranquilla en la costa Caribe e Iquitos en la selva amazónica peruana, como importantes centros de explotación en Suramérica (BAPTISTE et al, 2007), Mike Tsalickis, antecesor de las celebridades del programa de televisión "Animal Planet" y el mayor comerciante de fauna en la ciudad, "compró en 1968, a indígenas locales 14.890 animales silvestres y vendió 10.921 repartidos así: 47\% de primates; 0,2\% de carnívoros; $52 \%$ de aves y $0,62 \%$ de serpientes" (BAPTISTE et al, 2007, p. 9). 
Nuestras fuentes orales, producto de conversaciones con los pobladores identifican este período como la época de las tigrilladas durante la cual se realizó una explotación masiva de pieles de jaguares (Panthera onca) y nutrias (Pteronura brasiliensis). También hay que resaltar que se realizó una importante extracción y comercialización de fauna silvestre que ha sido poco documentada. Solo para citar un ejemplo, en 1957 Mike Tsalickis y Max Oldenburg crearon la sociedad Aero Tarpon Zoo Ltda., hoy en día localizado en Tarpon Spring, 45 minutos al norte de Tampa la parte del golfo de México en la Florida, quienes con un hidroavión Catalina viajaban hasta Tarapacá, Pedrera, Mitú y Araracuara, trayendo especies silvestres a la ciudad de Leticia y luego las embarcaban rumbo a la ciudad de Tampa y Miami - USA 6 .

El escenario nacional y local anteriormente descrito posibilitó el inicio de la comercialización de pescado a mediados del siglo XX en la ciudad de Leticia, gracias a la apertura de nuevas líneas comerciales tanto aéreas como fluviales. Así se originó una estrecha relación entre la historia de la extracción de fauna y pesca con fines comerciales con la historia del transporte en la selva amazónica colombiana (RODRÍGUEZ, 1991, 39), al mismo tiempo que emparenta la inversión del Estado en infraestructura como condición de desarrollo del sector privado. Leticia fue conectada con el país a través de la aviación, que a su vez sirvió para enviar animales silvestres, pieles y otros productos de la selva a Bogotá. Con ello se incrementó del número de militares y policías en la ciudad, se realizaron mejoras en los sistemas de comunicación, en donde la actividad privada fue acompañada de inversión pública, evidenciando una rápida modernización de la ciudad a mediados del siglo XX, lo que sentó las bases para convertirse en una importante ciudad comercial, junto con su ciudad "gemela" Tabatinga, sobre el río Amazonas, en medio de Iquitos (Perú) y Manaos (Brasil).

Desde entonces, el presupuesto del gobierno central ha sido un importante soporte económico de la ciudad que ha fortalecido las actividades comerciales, de una población que demanda bienes de consumo provenientes del interior del país y de los países vecinos, contribuyendo a la activación del comercio por vía fluvial en el transporte de alimentos y materiales de construcción y poco a poco el sector privado empieza a dinamizar algunos sectores económicos, siendo el envío de pescado a Bogotá, uno de los factores clave de esa dinamización.

${ }^{6}$ Palabras de Mike Tsalickis, propietario de la empresa Tarpoon Zoo. Entrevista concedida a Germán Palacio en Tampa - Florida, 13 de marzo de 2013. 


\section{INDUSTRIA PESQUERA Y FACTORES CLAVE PARA COMPRENDER LA IRRUPCIÓN DE LETICIA EN EL MERCADO DE PESCA CONTINENTAL (FLUVIAL) ${ }^{7}$}

Si bien la comercialización de pescado de Leticia a Bogotá es decisiva para entender el desarrollo de la ciudad, no es autoevidente que haya empresarios que se quieran arriesgarse enviándolo en avión sino hasta cuando se dieron las condiciones logísticas y de mercado que abrieron esa oportunidad. En lo que sigue se tocan 4 factores que pueden explicar el aprovechamiento de un nicho de mercado desde Leticia y que se describen a lo largo del texto: (I) la demanda de pescado a nivel nacional, (II) la abundancia de las especies explotadas, (III) la disponibilidad tecnológica para la pesca y (IV) la conservación y transporte del pescado.

A grandes rasgos lo que ocurre puede ser visto así: gran crecimiento de la pesca continental desde los años 50's que se ha mantenido constante debido a aparentes mejoras tecnológicas que pueden ser vistas como desmejoras para el medio ambiente. Es importante aclarar que los estudios consultados reconocen que los reportes de datos -nacionales o regionales - de pesca continental, pueden ser insuficientes e incompletos por la dispersión o falta de información, por lo que hasta el momento las estadísticas pesqueras existentes corresponden a los desembarques de pescado en los principales puertos, "dejando por fuera las pesquerías de subsistencia y las de comercio local" (VALBO-JORGENSEN; SOTO; GUMY, 2002, p. 2).

Para comprender mejor como se abrió la oportunidad para Leticia, la pesca continental en América Latina ha presentado un crecimiento constante, como se registró en el año 2004 con el desembarque de 552.000 toneladas, correspondiente al 5,7\% de las capturas continentales mundiales. En Latinoamérica, el 90\% del total de estas capturas se concentraba solo en seis países; Brasil: 45\%; México: 18\%; Venezuela: 9\%; Perú: 7\%; Argentina: 6\%; Colombia: 5\% (VALBOJORGENSEN; SOTO; GUMY, 2002, p. 3).

\footnotetext{
Para plasmar este trabajo se consultó una extensa bibliografía secundaria compuesta por estudios de investigación, técnicos, biológicos, proyectos institucionales y tesis, relacionados con la actividad pesquera en la región. También se revisaron archivos públicos y privados, tales como la Biblioteca del Banco de la República sucursal Leticia, Biblioteca Nacional de Colombia, Boletín Estadístico Pesquero - INPA, Gobernación de Amazonas, Alcaldía de Leticia, SINCHI y Cámara de Comercio del Amazonas. Se realizó la revisión de periódicos de circulación local y nacional: La hoja parroquial (1955-1970), periódico Anaconda (1988-2008), El Espectador y El Tiempo (1968-1980). Se realizaron una serie de entrevistas a investigadores académicos en la región, a funcionarios de la Aeronáutica civil, de la Cámara de Comercio del Amazonas, y de la oficina de rentas de la Alcaldía de Leticia. Se entrevistaron comerciantes locales (venta de motores, implementos para la pesca y combustibles), pescadores, intermediarios, acopiadores y transportadores de carga en la ciudad de Leticia, Tabatinga (Brasil), Santa Rosa y Caballo Cocha (Perú) y se realizaron dos visitas a la Plaza de mercado de Paloquemao en la ciudad de Bogotá.
} 
Los dos primeros países son los responsables del significativo aumento de la producción, y en el caso de Brasil, se explica por el aumento de los desembarques de la pesca industrial en el delta amazónico, mientras que, para México, por la repoblación de embalses.

Algunos autores se han referido a la crisis global de la pesca. Es importante tener en cuenta este factor ya que permitiría que Leticia en un momento dado llene un nicho de mercado. En el caso específico del medio Amazonas, es decir, lugar en que suele ubicarse Leticia y Tabatinga, entre Manaos e Iquitos, el cuadro no se puede ver como una réplica simple o mecánica de la crisis global de la pesca. Esta crisis se debe ver de manera escalonada, de modo que se presenta primero en algunos lugares y así son buscados otros para sustituirlos. Aunque todavía puede ser cierto que en el imaginario de las poblaciones de pescadores amazónicos prevalece la idea de que el recurso pesquero es inagotable, esto se debe a que el medio Amazonas ha sido foco más reciente de comerciantes de pescado que en otros lugares. Evidentemente la explotación excesiva disminuye la pesca al punto de desaparecer a las especies de mayor importancia comercial (DÍAZ, 1993), generando no solo un daño ambiental en la cadena trófica ${ }^{8}$, sino también consecuencias económicas y sociales para las familias que dependen exclusivamente de la actividad pesquera (MOJICA, 2002).

En consecuencia, uno de los factores macro que se debe tener en cuenta es la "crisis pesquera" global y su relación con el consumo de pescado en Colombia primero y luego en la Amazonia. Los estudios pesqueros existentes en Colombia nos permiten identificar problemas en común en las pesquerías continentales e incluso realizar comparaciones sobre el proceso de explotación pesquera entre algunas cuencas nacionales, por ejemplo, la cuenca del río Magdalena, el Sinú y el Amazonas. En general, podemos afirmar que, a raíz del incremento de la demanda del mercado nacional desde mediados del siglo XX, se introdujeron en estas cuencas nuevos artes de pesca y se mejoró en algunos casos la capacidad de almacenamiento y transporte, pretendiendo alcanzar una semiindustrialización de la actividad, aunque prevalecen los métodos tradicionales de pesca; esto trajo consigo el aumento de la explotación de las especies en las cuales se ha concentrado la demanda. Sin embargo, a mediano y largo plazo, este "mejoramiento" tecnológico generó una disminución de las poblaciones existentes, e inició la explotación de otras especies que antes carecían de valor económico. Podemos pasar así a presentar la situación del consumo de pescado en Colombia, que ha dado lugar a convertir el Medio Amazonas como un lugar

8 Generalmente se explotan las especies de mayor tamaño que se encuentran en la parte alta de la cadena trófica, tal como sucede con los bagres en la región amazónica. 
importante para la pesca y el efecto consiguiente que presenta este artículo en términos del desarrollo y transformación urbana de Leticia.

\section{EL CONSUMO DE PESCADO EN COLOMBIA}

Se suele considerar que en Colombia el consumo de pescado es bajo. Con el fin de contextualizar esta afirmación, según el último estudio de 17 de Agosto de 2016 de la Organización de las Naciones Unidas para la Agricultura y la Alimentación - FAO, el consumo per cápita de pescado en Colombia es de aproximadamente 6 kilos por año, muy por debajo del crecimiento mundial 20 kilos per cápita/año, y de la media latinoamericana 9 kilos per cápita/año (FAO, 2016). Una de las razones es histórica. El poblamiento colombiano se concentró en los Andes, casi desde la Colonia y el período Republicano. La capital y los ejes de poblamiento más importantes estuvieron en los Andes y la costa. En la costa Caribe, las ciudades coloniales, Cartagena, Santa Marta y Riohacha se estancaron durante la República y sólo Barranquilla llegó a ser importante desde fines del siglo XIX, al tiempo que el eje del río Magdalena se constituye en la clave de la nacionalidad en la primera parte del siglo XX. Así la pesca comercial se concentró en el río Magdalena. Los bogotanos aprendieron a comer viudo de pescado, bagres principalmente cuando descendieron del altiplano a tierra caliente desde mediados del siglo XIX (PALACIO, 2006). La pesca comercial en Colombia se ha concentrado en el río Magdalena cercano a los principales mercados, en particular, Bogotá, y hasta hace poco no se contabilizaba la rica cuenca orinocense.

Desde la década de 1930 se empezaron a gestar iniciativas para impulsar la industria pesquera y promover el consumo de pescado a nivel nacional, sustentadas en ideas nutricionales como las del intelectual antioqueño Luis López de Mesa $^{9}$, quien exhortaba sobre el atraso de la civilización y la raza colombiana, generado en buena medida por la carencia de fósforo y calcio. Se consideraba importante suplir esas carencias alimenticias a través del consumo de pescado, ya que este recurso contiene altos niveles de fósforo y calcio, se evitaba “el problema de la degradación racial asociada al estancamiento del país en su camino hacia la civilización y el progreso" (CAMARGO, 2009, p. 179). En la época se refleja

\footnotetext{
"Humanista antioqueño, estudio medicina en la Universidad Nacional (1912), se especializó en psiquiatría y psicología en la Universidad de Harvard. En 1934 a través del Ministerio de Educación impulsó un programa para mejorar la instrucción académica en los establecimientos de enseñanza y a la vez, transformar el ámbito social y cultural de la comunidad, revolucionando sus hábitos alimenticios, de vivienda y de vestuario. A través de sus numerosos ensayos y publicaciones durante las décadas de 1930’s y 1940’s expresó su proyecto civilizador, proceso que incluía según él, raza, industria, cultura y misión histórica” (GÓMEZ, s.f.)
} 
el incremento de la producción pesquera que para el año de 1938 era de 10 toneladas, pasando en 1965 a 53 toneladas (MOLANO; ACERO, 1967).

Finalizando la década de 1950 se realizaron estudios a nivel nacional y regional para intentar medir las potencialidades pesqueras del país concentrándose en el bajo consumo de peces y en el subdesarrollo de la actividad, tanto tecnológico como comercial. Algunos de estos estudios fueron apoyados por la Organización de las Naciones Unidas para la Agricultura y la Alimentación - FAO (CAMARGO, 2009). En 1962, el Estado apoyó la promoción del sector pesquero declarándolo como una industria básica y, como tal, exenta de impuestos de renta en un 100\% hasta 1969 (ASOCIACIÓN NACIONAL DE INDUSTRIALES, 1971, p.15). Sujeto a otras presiones en los años 70’s, el Estado autorizó la importación de productos pesqueros al país, provenientes de los países del Pacto Andino, generando un impacto negativo en la naciente industria pesquera (BELTRÁN; VILLANEDA, 2000), causado en gran medida por la incapacidad económica de esta nueva actividad para competir con países ricos por su posición en el Pacífico y ser bendecidos en términos de riqueza pesquera por la corriente de Humboldt, tales como Perú y Ecuador.

El fomento del consumo de pescado en el país desde mediados del siglo XX no estuvo acompañado de una reglamentación necesaria para informar y capacitar a los pescadores sobre la importancia de la conservación de las cuencas, debido, no tanto a una presencia estatal débil, sino que nuestra comprensión contemporánea sobre población y mercados crecientes con recursos limitados no existía en la época. Así, se desencadenó una explotación excesiva de los recursos pesqueros generando sobrepesca, deterioro y alteración principalmente de las cuencas, tras la utilización de la dinamita para la pesca y la contaminación por vertimientos de aguas industriales, domésticas y agropecuarias que junto con la minería alteraron el hábitat de los peces y generaron una disminución de las poblaciones.

Es importante entender que durante este período se realizaron modificaciones tecnológicas en los aparejos o artes de pesca existentes en el país. A modo de ejemplo, en la cuenca del Magdalena se modificó el chinchorro y la atarraya, aumentando la capacidad de explotación de las mismas, dando como resultado el surgimiento de las chinchorradas, los trasmallos y atarrayas modificadas -huequeras o rastreras (GUALDRÓN, 2002). En la actualidad está prohibida la utilización de estos artes de pesca en caños y ciénagas ya que, al no ser selectivos, afectan las poblaciones juveniles y comprometen la sostenibilidad de las especies.

Las limitaciones en el transporte y la deficiencia en las técnicas de 
conservación del recurso pesquero existentes durante la primera mitad del siglo, no hacían fácil la comercialización, manteniéndose bajos niveles de consumo per cápita. Pero una década después, tras la mejora en los medios de transporte, se suplió la demanda hacia las principales ciudades del país y comenzó a ejercerse una mayor presión pesquera que conllevó, para el caso de la cuenca del Magdalena, una disminución de la producción pesquera, generada no sólo por la explotación de este recurso, sino también por factores antrópicos anteriormente mencionados, tales como la contaminación por vertimientos de aguas industriales, domésticas, agropecuarias, la minería, entre otras (MOJICA, 2002, p. 36).

Poco a poco se desdoblan los tipos de pesca: la pesca industrial y la pesca artesanal. Mientras que la pesca industrial es principalmente marítima y proporciona "las mayores capturas, goza de las mejores inversiones y cuenta con infraestructura de pesca más moderna” (LASSO; MORALES, 2011, p. 13); la pesca artesanal es desarrollada por los pescadores de manera parcial o de tiempo completo, obteniendo una gran parte de sus ingresos anuales de esta actividad. Los productos que extraen son comercializados a nivel local o en puntos de acopio, y los pescadores artesanales se caracterizan por tener un bajo nivel económico y aprendieron el oficio por tradición familiar desde la juventud e incluso desde la infancia.

El pescado era prohibitivo en términos de costo, aunque la celebración de los días santos (Semana Santa) daban un pequeño impulso al consumo de pescado por razones religiosas. Los precios de los productos pesqueros en las principales ciudades eran altos, en comparación con otros productos cárnicos (vacuno, avícola y porcino).

La demanda de peces de aguas continentales, desde la segunda mitad del siglo $\mathrm{XX}$, se ha concentrado en especies tales como: bocachico (Prochilodus magdalenae), cachama/gamitana (Colossoma macropomum) y bagres de río (Pseudoplatystoma magdaleniatum, Pseudoplatystoma metaense, Pseudoplatystoma punctifer, Pseudoplatystoma tigrinum). Los bagres son los preferidos por el consumidor de los valles calientes, particularmente del Magdalena y de las ciudades del altiplano, ya que argumentan que tienen un buen sabor, por su rápida y fácil preparación, además de tener un hueso central sin espinas. Algunos les han atribuido propiedades afrodisíacas ${ }^{10}$.

El consumo de pescado varía de región a región y está determinado en buena medida por los precios y la cercanía a los centros de acopio, siendo mayor el consumo en las poblaciones ribereñas de aguas continentales y costeras, y en

10 Afirmaciones que expusieron los compradores de pescado en la plaza de mercado de Paloquemao ubicada entre la calle 19 con carrera 24 en la ciudad de Bogotá, como parte de las actividades de campo de la presente investigación. 
las principales ciudades como Bogotá, Cali, Medellín, Cartagena, Barranquilla, Bucaramanga y Villavicencio. La diferenciación de las especies consumidas también obedece a las condiciones económicas de la población, tal como lo exponen Claudia Beltrán y Abraham Villaneda (2000) definiendo el perfil de pesca y acuicultura en Colombia, así:

Las personas de estratos económicos medio y alto tienen mayor propensión al consumo de filetes, pescado fresco de mar y cultivo, mariscos [...] y productos de valor agregado. La gente de menores ingresos se inclina por los pescados de aguas continentales (bocachico, bagre, nicuro, doncella, capaz, pescadilla), pescado seco-salado (BELTRÁN; VILLANEDA, 2000, p. 7).

Dejando de lado la idea curiosa de "propensión", como si fuera solo un asunto de "gusto" y no de otras condiciones, incluidos precios, el consumo de pescado ha aumentado en buena medida por el reconocimiento internacional otorgado a sus altas cualidades nutricionales ${ }^{11}$, sin embargo, el desconocimiento del consumidor colombiano sobre las variedades de especies disponibles y sus modos de cocción o preparación (se limitan a frito y sudado), sigue siendo una limitante en el momento de comprar pescado ${ }^{12}$.

\section{CONSUMO Y COMERCIALIZACIÓN DE PESCADO EN LA AMAZONIA COLOMBIANA}

La pesca es una de las actividades más antiguas de la Amazonia. Sus pobladores han desarrollado esta actividad desde tiempos remotos y poseen un invalorable conocimiento de los recursos pesqueros existentes en la cuenca y de cómo la abundancia y escasez de pescado está regulada por el pulso de inundación del río. Aunque la yuca (mandioca) sea el producto clave de la alimentación, y la cacería sea otra actividad importante, el pescado les proporciona la mayor parte de la proteína animal que consumen. Esto, sumado a la facilidad de acceso al recurso y a las grandes cantidades de pescado que pueden capturar, ya que la cuenca amazónica contiene la gran riqueza íctica en términos de volumen y de biodiversidad en el mundo (ALMEIDA; HOCHACHKA, 1995).

Algunos autores han descrito a los pobladores ribereños amazónicos como O povo das águas (PORRO, 1995), describiendo cómo el río ha definido la vida de

11 En el caso de los bagres "contienen aproximadamente 120 calorías por cada porción de 31/2 onzas. Igual que la mayoría de mariscos, este pescado contiene menos calorías por porción que aves o carne de cerdo. El bagre contiene también poco sodio" (LADEWIG; LOGAN, 1993).

12 Afirmaciones que expusieron los compradores de pescado en la plaza de mercado de Paloquemao ubicada entre la calle 19 con carrera 24 en la ciudad de Bogotá, como parte de las actividades de campo de la presente investigación. 
estos pobladores y sus actividades, resaltando cómo éstas varían siguiendo el pulso de inundación (aguas altas, aguas en descenso, aguas bajas y aguas en ascenso) y determinando las actividades de agricultura, caza, pesca y la extracción de productos del bosque (KVIST; NEVEL, 2002, p. 8). Carlos Witkoski (2007) describe cómo el conocimiento de los ribereños está basado en un tiempo ecológico y no cronológico, en la medida que mantiene su condición de frontera del capitalismo, como un patrón adaptativo de los amerindios en respuesta a los pulsos de inundación del nivel de las aguas que puede variar anualmente entre 8 a 15 metros.

La pesca puede ser considerada como una actividad con alguna dificultad de aprender, aunque demanda mucho tiempo y dedicación; los niños desde muy temprana edad están acompañando a sus padres en las jornadas ya que es, en ese espacio, en la práctica de la actividad que se inicia la transmisión del conocimiento, siendo este principalmente masculino.

Durante los últimos cincuenta años, en Leticia y su conurbación, Tabatinga, se han desarrollado varios tipos de pesca: comercial, semi-comercial, ornamental y de subsistencia; igualmente se han perfilado dos tipos de mercados bien definidos: el mercado local y el mercado hacia el interior del país. El mercado local se abastece principalmente de pescado de "escama", que es comercializado por pescadores locales e indígenas que viven principalmente en la ribera del río y sus afluentes próximos a la ciudad; existe una resistencia cultural al pescado de cuero ya que, son considerados pescados "reimosos"13. Especies como la sabaleta (Brycon sp.), la gamitana (Colossoma macropomun) y el pirarucú (Arapaima gigas), entre otras, eran consideradas como especies de primera categoría hasta por lo menos mediados del siglo XX, mientras que bagres como el rayado (Psendoplatystoma fasciatum), el dorado (Brachyplatystoma flavicans), y el lechero (Brachyplatystoma filamentosum) para la misma época eran especies de tercera categoría, por ser considerados "reimosos", en dicho regional, y casi no se comercializaban en el mercado local. Después de la década de 1960 se incrementó la demanda sobre estas últimas especies, clasificándolas como peces de primera categoría debido a su importancia económica, es decir, con demanda en el mercado en Bogotá, mas no de consumo entre la población local, ya que muchas de las creencias o restricciones culturales prevalecen hasta la actualidad, al igual que la preferencia de la población amazónica por los peces de escama.

La cantidad promedio de pescado que consume un habitante de Leticia está alrededor de 15 kilos por mes (PRIETO, 2006, p. 83), en parte por la facilidad

\footnotetext{
13 Un pez reimoso no puede consumirse porque puede generar algunos problemas de salud y dentro de algunos grupos étnicos se restringe su consumo a personas que tengan heridas abiertas o mujeres que se encuentren en estado de embarazo. Consultar: ZORRO, CHUMBE e CASTRO (1996), MORA (1985).
} 
de conseguir el pescado fresco y a menor precio que otros productos como la carne de res (SALINAS, 1994). Sin embargo, muchos pescadores reconocen que la composición y tallas de las capturas han cambiado en las últimas dos décadas. Esto puede estar cambiando aceleradamente por el desarrollo del turismo en la última década ya que presiona al alza el recurso por la demanda de una población del país y del exterior que llega en vuelos comerciales desde Bogotá.

Durante los años 1950's, se inició la comercialización de pescado hacia Bogotá y al no existir el servicio de energía eléctrica en la ciudad de Leticia, la única forma para conservar el pescado era a través del sistema seco-salado (TELLO, 2002). Este proceso era adelantado en la gran mayoría de veces por el pescador. La comercialización del pescado seco-salado dio inicio a la modificación de las faenas de pesca y cambió las categorías sociales y económicas de algunas especies de peces, mencionadas anteriormente.

Los primeros compradores o comerciantes de pescado seco-salado en Leticia, fueron Hernando Quintero Uribe y Juan Domingo Rodríguez (apodado gringo negro); posteriormente Mike Tsalickis y Max Oldenburg. Durante los años 1950's los comerciantes iniciaron el desarrollo de un sistema de endeude aún incipiente que se fortaleció en la siguiente década, adelantándole parte del pago por el pescado al indígena o colono pescador, que era básicamente sal ( $\mathrm{NaCL}$ ) para la conservación del pescado y algunos otros productos de insumos para la pesca, tales como, las cuerdas de algodón y anzuelos. A través de este sistema podían garantizar que ese pescador les vendiera a ellos el producto de la faena y no a otros comerciantes (TELLO, 2002).

Durante esta época los pescadores utilizaban pequeñas embarcaciones impulsadas a remos, y el tiempo de dedicación a esta actividad era parcial (PINTO; SANTOS, H.; WITKOSKI, 2007), no existía aún la figura del pescador profesional o "especialista", que realizaba la pesca como una actividad exclusiva, sino que se trataban de pescadores-agricultores, que comercializaban los excedentes de la pesca en los centros poblados más próximos a su comunidad o vivienda, permitiéndoles acceder a bienes de consumo básico.

Las poblaciones indígenas utilizaban el anzuelo, el arpón y la flecha como los principales utensilios de pesca, pero al iniciarse la pesca comercial, se popularizó el uso de la cuerda, el espinel, malla hondera y estacionaria, que permitieron obtener más fácil los peces, generando una diversificación de los artes y métodos pesqueros que no dejaban de ser artesanales. La mediana tecnificación que se dio en cuanto a artes de pesca y tipos de embarcaciones, no logró prescindir de los métodos tradicionales, sino por el contrario enriqueció la actividad, garantizando la explotación durante todo el año (RODRIGUEZ, 1991, p. 54). 
Durante los años 50’s, cuando inició la pesca comercial, la infraestructura de acopio era limitada, con una baja capacidad de almacenamiento. La pesca comercial se incrementó con la instalación de cuartos fríos en la ciudad y se conoce localmente como la llegada del hielo. En 1958 se instaló el primer cuarto frío con una capacidad de almacenamiento de 5 toneladas, para su funcionamiento se utilizaron dos plantas eléctricas de ACPM al no existir en la ciudad el servicio de energía eléctrica (SALINAS, 1994, p. 62). Hasta ese momento todo el pescado comercializado de cuero o escama que era llevado a las ciudades del interior del país era seco-salado, siendo la única forma disponible de conservación.

La instalación de los cuartos fríos, permitió ampliar el sistema de conservación y la capacidad de almacenamiento de pescado en la ciudad. Los acopiadores impulsaron localmente la pesca comercial a través de los adelantos, como mecanismo de negociación para atraer a los pescadores, junto con el ya conocido sistema de endeude; el uso de uno de estos dos vínculos estaba asociado a la capacidad de captura del pescador. El adelanto es un sistema donde el acopiador le entrega cierta cantidad de dinero, materiales o equipos de pesca al pescador para que éste realice sus actividades, y el costo total de dichos materiales, será descontado inmediatamente en el pago por la compra del pescado que logre capturar. El sistema de endeude, de la misma manera que en el anterior, se le entrega al pescador dinero, materiales o equipos de pesca, pero el pago de la deuda se hará a través de un porcentaje que se va descontando durante las siguientes faenas de pesca (FEDERACIÓN COLOMBIANA DE ACUICULTORES, 2001). Estos acuerdos se realizaban verbalmente con pescadores brasileros, colombianos y peruanos.

Los adelantos, durante las décadas de 1960 hasta 1980, consistieron en bloques de hielo, neveras de icopor y combustible, junto con la posibilidad de financiación, bajo el sistema de endeude, de motores de baja potencia ( 9 - 40 HP). Estos vínculos comerciales o formas de negociación le permitieron al acopiador garantizar el suministro de pescado, vincular a más personas y ampliar el área de explotación gracias al uso de los motores diésel (McGRATH, et al, 1993). Este sistema generó monopolios y dependencias de los pescadores a los grandes acopiadores ubicados en el puerto de Leticia.

En 1974 se promovió el ensanchamiento del aeropuerto, a fin de permitir el aterrizaje de aviones más modernos, con una capacidad de carga mayor y reduciendo el tiempo de vuelo de seis horas Bogotá - Leticia a solamente tres. Se destinaron 29 millones de pesos para esta obra que amplio la pista a 1.900 metros (CÓRDOBA, 1972). La ampliación dinamizó el transporte aéreo ya que posibilitó el ingreso de nuevas aerolíneas con flotas de aviones de mayor 
capacidad, que no podían operar anteriormente en la ciudad, pasando de dos vuelos semanales con una capacidad de carga entre 4 y 5 toneladas, a diez vuelos en aviones Cutis C-48 y tipo Jet con capacidad de carga de hasta de 10 toneladas, debido a la gran cantidad de pescado que se acumulaba rápidamente en las bodegas. Las empresas aéreas que operaron hacia Leticia en ese entonces fueron: ACES, Intercontinental de Aviación, AIRES, Avianca, Satena, Taxi Aéreo Opita (TAO), Aerotal, Aerotaxis y Aeropesca.

La prohibición de la comercialización de fauna silvestre, tras la entrada en vigor del Código Nacional de Recursos Naturales (Decreto Ley 2811 de 1974) y de Protección del Medio Ambiente (Decreto Ley 1608 de 1978), permitió que finalizando la década de 1970's, la ciudad empezara a contemplar el turismo como una alternativa económica; pero también, generó un crecimiento acelerado de la comercialización pesquera, debido a que los comerciantes de fauna silvestre se convirtieron en acopiadores de pescado, dinamizando e inyectando capital a esta actividad.

En 1963 el pescado movilizado desde Leticia a la ciudad de Bogotá por vía aérea fue de 1.200 toneladas (CORDINI; PLATA, 1963); en 1984 se reportó el envío de 4.260 toneladas (BANCO DE LA REPÚBLICA, 1984), incrementándose la actividad en un $400 \%$ en 20 años. Posteriormente continuó ascendiendo el volumen de comercialización de pescado, pero a una tasa más lenta reportando en 1989 la comercialización de 5.756 toneladas (BANCO DE LA REPÚBLICA, 1991).

El incremento de esta actividad generó en la ciudad empleos directos e indirectos, que conllevaron a la formación y consolidación de asociaciones y sindicatos, cuya actividad está estrechamente relacionada con la pesca comercial, tales como el sindicato de Braceros ${ }^{14}$, la Asociación de Propietarios de Transporte Público de Carga Amazonas, la Asociación de Expendedores de Combustibles Asociados - AXCOA.

Los registros mercantiles del departamento de Amazonas, muestra que el $93.8 \%$ de los establecimientos que existen en la ciudad de Leticia, son de carácter comercial, tales como la venta de víveres, abarrotes en general, restaurantes, compra y venta de pescado, venta de prendas de vestir, calzado, etc. Caracterizando a Leticia como una ciudad distribuidora de productos de consumo final y de servicios, en donde la comercialización de pescado asume el mayor dinamismo (ACOSTA et al., 1997), moviendo grandes cantidades de dinero, junto con la comercialización de combustibles y el transporte de carga.

14 Los braceros son los cargadores de pescado, quienes descargan de las lanchas el pescado y los trasladan a los cuartos fríos. 
Desde 1974, cuando se creó la Cámara de Comercio del Amazonas hasta el año 2008 se realizaron más de 200 registros vinculados a la pesca comercial.

Sin duda alguna, Leticia se ha convertido en un importante centro de acopio pesquero, que para el año de 1987 contribuía con el 16\% del total de la producción nacional (CASTRO, 1992, p. 265) y para el 2001 pasó al 38\% (CAMACHO, 2006, p. 8), presentando un incremento de más del 50\% en 14 años y contribuyendo cada vez más al total del volumen explotado. La pesca se concentra en la explotación de los bagres, que es el nombre común que se le da a las especies que pertenecen a la familia Pimelodidae. Actualmente la Amazonia colombiana es la segunda cuenca a nivel nacional de producción pesquera en aguas continentales, después del Magdalena y la comercialización de pescado sostiene parcial o totalmente las economías de las poblaciones locales asentadas en la ribera del río Amazonas y sus afluentes. En la Amazonia colombiana no se desarrolla una pesca industrial ya que no se cuenta con una flota de barcos, ni con la infraestructura para poder tener esta clasificación, a diferencia de otras zonas de la cuenca amazónica, siendo la más cercana a Leticia la ciudad de Manaos (Brasil). La tecnificación de la actividad a través de los motores, las neveras de "icopor" y el hielo, les ha permitido a los pescadores aumentar el volumen de peces y el radio de explotación de los mismos, yendo cada vez a áreas más distantes.

El surgimiento y desarrollo de la comercialización pesquera en Leticia estuvo acompañado de acciones estatales y privadas que generaron trasformaciones regionales, acompañadas de un discurso local de modernización que se evidencia en la prensa local desarrollado por los comerciantes ${ }^{15}$. Los estudios sobre pesca y comercialización de pescado en la Amazonia colombiana provienen de biólogos. Sin embargo, cada vez es más común encontrar investigaciones que incursionan en la descripción de aspectos sociales y culturales como parte complementaria de estos trabajos, dejando ver una estrecha relación entre las sociedades amazónicas y el recurso pesquero (CAMACHO, 2006; SALINAS; AGUDELO, 2000, RODRÍGUEZ, 1991). O, mejor, dimensiones culturales se amarran de aspectos de transformación económica. La pesca comercial ha generado cambios y trasformaciones en el medio Amazonas en los últimos 50 años (PEREIRA, 2007, p. 172). Lo cual incluye una considerable transformación de la ciudad de Leticia.

15 El concepto de modernización es erróneamente utilizado en la época ya que se confundió con el de modernidad. Modernización es utilizado para entender la separación Estado-Iglesia, entre otros procesos y no se refiera al desarrollo tecnológico- industrial como si lo es modernidad. Consultar BERQUIST (1986). 


\section{REFLEXIONES FINALES}

Leticia se convirtió en centro de acopio pesquero importante, y como producto de la necesidad del Estado colombiano de apropiar su frontera sentó las bases de la colaboración y encabalgamiento del sector público y el privado desde la década de 1950. Dentro de este proceso se generaron dinámicas locales y regionales que permitieron comercializar toneladas de pescado en el mercado de Bogotá, principalmente, provenientes del Trapecio Amazónico y sus alrededores, Brasil y Perú, ya que Colombia solo posee 116 kilómetros sobre la margen del río Amazonas. Este artículo evidencia cómo la pesca con fines comerciales se convirtió en un eje trasformador de las dinámicas sociales y económicas regionales consolidando a su capital, Leticia como centro urbano importante en una realidad multinacional, desde antes de que el Estado, con los procesos de descentralización de fines de 1980s y la Constitución política de 1991 haya dado un salto cualitativo para iniciar un proceso de conversión de su frontera en una región con autoridades departamentales y municipales elegidas por votación popular. Estas autoridades de un departamento con un capital humano con una baja formación profesional han batallado con muchos problemas que resultan en la destitución de sus mandatarios que a su vez tienen muy poca capacidad de gestión en la maraña alambicada del sistema político e institucional colombiano. Mucho tiempo de ser colonia interna, requiere a su vez mucho tiempo de formación de talento humano bien calificado para afrontar los desafíos institucionales en el siglo XXI.

Otros desarrollos investigativos deben abocarse a nuevos desafíos de la pesca comercial en la Amazonia en un contexto que está cambiando rápidamente. Primero, el incremento poblacional de las ciudades amazónicas, especialmente, Iquitos (Perú) y Manaos (Brasil) que en las últimas dos décadas están compitiendo como centros de acopio pesquero en el medio Amazonas, cambiando las dinámicas de comercialización existentes. Segundo, el incremento en los costos de la actividad (combustible y energía eléctrica) en Leticia, hace cada vez más inviable la pesca comercial, conllevando al cierre de cuartos fríos, y por último el incremento en el mercado nacional de bagres asiáticos que se consiguen a menor precio en las principales plazas de mercado en el país, afectando la comercialización pesquera en Leticia.

Por último, la relación entre la ciudad y la pesca puede servir de elemento clave para el análisis de las ciudades pequeñas y medianas en el medio Amazonas. En este caso, el acercamiento a la explotación pesquera en la ciudad de Leticia. Igualmente, nos puede llamar la atención sobre la necesidad no solamente de 
la armonización normativa en ciudades del Estado do Amazonas en Brasil, el departamento de Amazonas en Colombia y el departamento de Loreto en Perú para lograr proteger la composición de las capturas que permitan mantener la competitividad y la sostenibilidad ambiental de la región.

\section{BIBLIOGRAFÍA}

ACOSTA, L. E. et al. Estructuras y dinámicas sociales, cultural y económica del Área P.A.T. En: Zonificación Ambiental para el plan modelo ColomboBrasilero (Eje Apaporis-Tabatinga: PAT). Santafé de Bogotá: Instituto Geográfico Agustín Codazzi (IGAC), p. 265-364, 1997.

ALMAIDA, M. F.; HOCHACHKA, P. W. Air-breathing fishes: metabolic biochemistry of the first diving vertebrates. Biochemistry and molecular biology of fishes. Vol. 5, p. 45-55, 1995.

ANGEOLETTO, F.; ESSY, C.; RUIZ, J. P. Ciudades medias de Brasil: una tipología socioambiental. Belém: Novos Cadernos NAEA. Vol. 19, n. 1, p. 175192, jan - abril 2016.

APONTE, J. Leticia y Tabatinga: transformación de un espacio urbano fronterizo en la Amazonia. Leticia: Universidad Nacional de Colombia Sede Amazonia, Tesis de Maestría, 2011.

Asociación nacional De Industriales. La Industria Pesquera en Colombia. Medellín: Asociación Nacional de Industriales -ANDI, 1971.

BANCO DE LA REPÚBLICA. Informe económico de la comisaría especial del Amazonas, 1989. Leticia: Banco de la República, seccional Leticia. 1991.

. Estudio Económico Regional. Leticia: Banco de la República, seccional Leticia. 1984.

BAPTISTE, L. G. et al. La fauna silvestre colombiana: una historia económica y social de un proceso de marginalización. Bogotá: Instituto de Investigación de Recursos Biológicos Alexander Von Humboldt. Disponible en: < http://www. humboldt.org.co/pdf/usoyval/Baptiste.pdf> Consultado: 03 mar 2007.

BELTRÁN, C. S.; VILLANEDA, A. Perfil de la pesca y la acuicultura. Subdirección de investigaciones. Santafé de Bogotá: INPA. Junio 2000. Pág. 1

BERQUIST, C. Los trabajadores del sector cafetero y la suerte del movimiento obrero en Colombia 1920-1940. En: SÁNCHEZ, G.; PEÑARANDA, R. (comp.). Pasado y presente de la violencia en Colombia. Bogotá: CEREC, p. 111-165, 1986. 
CAMACHO, K. A. La pesca del bagre pintadillo rayado Pseudoplastytoma fasciatum (Linnaeus, 1766): Aspectos del conocimiento local, de la biología pesquera y de los parámetros poblacionales en el alto río Amazonas (sector de Leticia-Colombia). Leticia: Universidad Nacional de Colombia Sede Amazonia, Tesis de Maestría, 2006.

CAMARGO, A. Una tierra bondadosa: progreso y recursos naturales en la región del río San Jorge, siglo XX. Bogotá: Historia Crítica, n. 37, p. 170-191, ene-abr 2009.

CASTRO, D. La pesca en la Amazonia colombiana. En: Amazonia Colombiana, diversidad y conflicto. Santafé de Bogotá: Ágora, 1992.

CORDINI, J. M.; PLATA, J. Estudio sobre la pesca en Leticia y consideraciones sobre la Extracción de piscicultura de Tota. Bogotá. Departamento Administrativo de Planeación. 1963.

CÓRDOBA, A. M. Estudio socio-económico del municipio de Leticia y una estrategia de desarrollo. Bogotá: Universidad de Bogotá Jorge Tadeo Lozano, 1972.

DONADIO, A. La Guerra con el Perú. Medellín: Hombre Nuevo Editores, 2002.

DÍAZ, Á. Ecología y pesca en Canarias: una aproximación histórica a la relación hombre-recurso. En: Historia y Ecología. Madrid: Editorial Ayer, n. 11, p. $207-$ $231,1993$.

ESCOBAR, J. Leticia - Alfredo Vásquez Cobo (SKLT / LET). En: El portal de la Aviación. 2010. Disponible en: <http://www.aviacol.net/aeropuertoscolombianos/leticia-alfredo-vasquez-cobo-sklt-let.html> Consultado: 3 mar 2016.

- Agencia Interamericana de Aviación - AIDA. En: El portal de la Aviación. 2008. Disponible en: <http://www.aviacol.net/historia-aviacion/ empresa-colombiana-de-aerodromos-eca.html> Consultado: 20 jul 2016.

FAO. El estado mundial de la pesca y la acuicultura. Roma: Organización de las Naciones Unidas para la Agricultura y la Alimentación -FAO, 2016.

FEDERACIÓN COLOMBIANA DE ACUICULTORES. Diagnostico estratégico de la cadena productiva regional de pesca blanca de agua continental del Amazonas. Bogotá: Federación Colombiana de Acuicultores, Informe técnico, 2001. 
GALVIS, G. et al. Peces del Medio Amazonas: Región de Leticia. Bogotá: Conservación Internacional, 2006.

GÓMEZ, L. López de Mesa, Luis. En: Biografías, Gran Enciclopedia de Colombia del Círculo de Lectores (s.f.) Disponible en: < http://www.banrepcultural.org/ blaavirtual/biografias/lopemesa.htm> Consultado: 13 abr. 2016.

GONZÁLEZ-PEÑA, M. L.; SAMACÁ, G. D. El conflicto colombo-peruano y las relaciones del Centro de Historia de Santander (CSH), 1932-1937. Historelo, Revista de historia regional y local. Vol. 4, n. 8, p. 367-400, Jul-Dic 2012.

GONZÁLEZ, F. E.; BOLÍVAR, I. J.; VÁSQUEZ, T. Violencia política en Colombia: de la nación fragmentada a la construcción del Estado. Bogotá: CINEP, 2002.

GUALDRÓN, M. I. Plan de manejo de los recursos ictiológicos y pesqueros en el río grande de la Magdalena y sus zonas de amortiguación. En: Ajuste del Documento Recursos Hidrológicos, Ictiológicos y Pesqueros en la Cuenca Magdalena - Cauca. Diagnóstico (caracterización) y Estrategias de Política para la formulación del POMIM. Bogotá: Autoridad Nacional de Acuicultura y Pesca (AUNAP). 2002.

KVIST, L. P.; NEVEL, G. Bosque de la llanura aluvial del Perú: ecosistemas, habitantes y usos de los recursos. Folia amazónica, Vol. 10, n. 1-2, p. 5-55, 2002. LADEWIG, K.; LOGAN, D. El bagre es delicioso y nutritivo. Southern Regional Aquaculture Center. SRAC Publication, n. 501, (s.f.) abr, 1993.

LASSO, C.; MORALES, M. Catálogo de los recursos pesqueros continentales en Colombia: memoria técnica y explicativa. Bogotá: Ministerio de Ambiente, Vivienda y Desarrollo Territorial; Instituto de Investigaciones de Recursos Biológicos Alexander von Humboldt, 2011.

McGRATH, D. et al. Fisheries and the evolution of resource management on the lower Amazon floodplain. En: Human Ecology. Vol. 21, n. 2, p. 167-195, 1993.

MORA, Y. Alimentación y Cultura en el Amazonas. Bogotá: Fondo de Cultura Cafetero. 1985.

MOLANO, J.; ACERO, A. Antecedentes y resumen de la industria pesquera en Colombia: contribución al estudio de la geografía económica de la pesca colombiana. Bogotá: Fundación Universitaria Jorge Tadeo Lozano, 1967. 
MOJICA, J. I. Las pesquerías de la cuenca del río Magdalena: Ejemplo a no repetir. En: Libro Rojo de peces dulceacuícolas de Colombia. Bogotá: Universidad Nacional de Colombia, Instituto de Ciencias Naturales, p. 35-41, 2002.

NAVIA, G. Conozcamos la selva amazónica. Leticia: Fondo Editorial Masayaki, 1994.

PALACIO, G. Leticia y el corazón de Suramérica: una urbe en la triple frontera amazónica. En: Cidades na Floresta. Sao Paulo: Annablume, 2008. Pág. 151 164.

. Fiebre de tierra caliente. Una historia ambiental de Colombia, 18501930. Bogotá: Universidad Nacional de Colombia-ILSA, 2006.

PANTEVIS, Y. A. Leticia memorias de frontera. Leticia: Gobernación del Amazonas, 2015.

- Construyendo la historia ambiental de Leticia a través de la pesca.

Leticia: Universidad Nacional de Colombia Sede Amazonia, Tesis de Maestría, 2013.

PEREIRA, H. A diversidade da pesca nas comunidades da área focal do projeto PIATAM. En: Comunidades de vida e uso dos recursos naturais. Manaus: EDUA, p. 171-195, 2007.

PICÓN, J. E. Transformación urbana de Leticia. Énfasis en el período 19501960. La construcción de una ciudad en la selva amazónica y en una región trifronteriza. Leticia: Cooteam, Creditos Parra, 2010.

PINTO, T. J.; SANTOS, H.; WITKOSKI, A. C. Comunidades Ribeirinhas Amazônicas, modos de vida e uso dos recursos naturais. Manaus: Universidade do Amazonas, 2007.

PORRO, A. Opovo das águas: ensaios de etno-história amazónica. Ptrópolis: Vozes, 1995.

PRIETO, E. F. Caracterización de la pesquería en las lagunas de Yahuarcaca (Amazonas, Colombia) y pautas para su manejo sostenible. Guanare: Universidad Nacional Experimental de los Llanos Orientales "Ezequiel Zamora", Tesis de Maestría, 2006.

RODRÍGUEZ, C. Bagres, Malleros y Cuerderos en el bajo río Caquetá. Bogotá: Tropenbos - Colombia. 1991. 
RUIZ, W. Recopilación de documentos de la Prefectura Apostólica de Leticia. Datos para la historia. Leticia: Prefectura Apostólica de Leticia, 2001.

SALINAS, Y.; AGUDELO, E. Peces de importancia económica en la cuenca amazónica colombiana. Santafé de Bogotá: SINCHI, 2000.

SALINAS, Y. Aspectos de la biología pesquera de las poblaciones de los grandes bagres en el sector colombiano del río Amazonas. Bogotá: Universidad Nacional Pedagógica, Tesis, 1994.

TELLO, G. Informe Técnico: Diagnóstico actualizado sobre tecnología de procesamiento, mercado, comercialización y economía pesquera en la cuenca compartida Colombo-Peruana del río Putumayo. Proyecto TPC/RLA/2802(A). Apoyo al ordenamiento de la pesca en el río Putumayo. Iquitos - Perú. INADE, FAO, PEDICP, SINCHI. 2002.

TSALICKIS, M. Mike Tsalickis, propietario de la empresa Tarpoon Zoo. Entrevista concedida a Germán Palacio. Tampa, Florida: 2013. 1 CD.

VALBO-JORGENSEN, J.; SOTO, D.; GUMY, A. La pesca continental en América Latina: su contribución económica y social e instrumentos normativos asociados. Roma: COPESCAL, Documento Ocasional n. 11, 2002.

WITKOSKI, A. C. Terras, florestas e águas de trabalho. Os camponeses amazónicos e as formas de uso de seus recursos naturais. Manaos: Universidad Federal do Amazonas, 2007.

ZÁRATE, C. Silvícolas, siringueros y agentes estatales: el surgimiento de una sociedad transfronteriza en la Amazonia de Brasil, Perú y Colombia, 18801932. Leticia: Universidad Nacional de Colombia, Instituto de Investigaciones Amazónicas (IMANI), 2008.

ZORRO, M.; CHUMBE, J.; CASTRO, O. Métodos tradicionales de conservación del pescado en la Amazonia: análisis y alternativas. En: Políticas pesqueiras nos países Amazônicos. Belem: Associação de Universidades Amazônicas, Universidade Federal do Pará. Série de Cooperação Amazônica, 1996. 
